\title{
Maternal genetics may contribute to autism-like traits in mice via inflammation
}

\author{
BY CHARLES Q. CHOI
}

2 JULY 2021

Women who are genetically predisposed to inflammation may be more likely to have children with autism traits, according to a new study in mice.

Prior work suggests that inflammation in a pregnant woman - due to a severe infection or an autoimmune disorder, for example - is associated with a slightly increased chance of autism in her child. The new study probed the role a woman's genes might play in such inflammation and fetal brain development.

"Our results suggest that maternal genetics alone can be a contributing factor in increasing the risk of autism spectrum disorder in the child," says lead investigator Charis Eng, chair of the Cleveland Clinic's Genomic Medicine Institute in Ohio. "These mothers would have genes that predispose them to increased inflammation just in response to the pregnancy itself, and not because of any drugs, toxins or infections."

The scientists focused on PTEN, a gene that encodes a tumor-suppressing protein that also helps shape neuronal connections. PTEN mutations account for as much as 2 percent of all autism cases and 17 percent of those in which the child has an unusually large head.

Eng and her colleagues developed mice with only one fully functional copy of PTEN instead of the usual two. They bred these mice with wildtype mice, resulting in some pups that had two fully functional PTEN genes and others that only had one.

More pups from female PTEN mice that were bred with wildtype males died in the first eight days after birth than those from wildtype females bred with PTEN males. And the pups of those PTEN mothers were more likely to have abnormally large heads and behaviors reminiscent of autism, such as repetitive activities and reduced sociability with unfamiliar mice, even if they had two fully functional PTEN genes. 


\section{Spectrum | Autism Research News}

https://www.spectrumnews.org

In addition, pups with only one functional PTEN gene and a wildtype mother had less prominent autism-like behaviors, which suggests that maternal genetics can also play a protective role against genetic predisposition, the researchers say.

\section{Interacting genes:}

Still, of all the pups born to model PTEN mice, those with just one functional copy of PTEN had more severe autism-like behaviors than their wildtype littermates, underscoring the influence of the pup's genes.

"We know that with genes linked with autism like PTEN or FMRP, you could have people with the same mutation of a gene, but only 20 to 50 percent of them will end up with autism, so either there is some gene-gene interaction or gene-environment interaction going on," says Bryan Luikart, associate professor of molecular and systems biology at Dartmouth University in Hanover, New Hampshire, who was not involved in the study. "These new findings point to both: a geneenvironmental interaction with some sort of inflammation and a gene-gene interaction between mothers and offspring."

Compared with wildtype female mice, PTEN female mice showed significantly lower levels of the inflammation-suppressing molecule IL-10 during pregnancy, and their fetuses had correspondingly lower levels of key immune proteins in their livers. In addition, those fetuses showed signs of neuron loss and less of a barrier to protect the brain from toxins and intruders, according to postmortem tissue analyses. The pups also had fewer mature neuron-supporting glial cells. The findings were published in June in Translational Psychiatry.

Future work should test other genes for their ability to induce inflammation during pregnancy, Eng says. Researchers should also pinpoint exactly how the disruption of IL-10 activity can affect brain cells, says Jaclyn Schwarz, associate professor of psychological and brain sciences at the University of Delaware in Newark, Delaware, who did not take part in this study.

Scientists might one day be able to predict inflammation during pregnancy from a woman's genes, Schwarz says. Clinicians might then be able to treat such inflammation with compounds that boost IL-10, Eng adds.

Cite this article: https://doi.org/10.53053/EXRK3922 